\title{
Recent research advances in European Rhinology
}

\author{
Valerie J LUND CBE \\ Emeritus in Rhinology, University College London \& Honorary Consultant ENT Surgeon, \\ Royal National Throat, Nose and Ear and UCL Hospitals, UK
}

Research in rhinology is alive and well in Europe both clinically and scientifically. In all aspects of the nose and sinuses, from allergy, inflammation and infection to neoplasia there have been many important advances in recent years. These have been intimately related to position papers and consensus groups such as ARIA $^{1}$ (Allergic Rhinitis and its Impact on Asthma), the European Position Paper on Endoscopic Management of Tumours of the Nose, Paranasal Sinuses and Skull Base ${ }^{2}$ and EPOS ${ }^{3}$ (European Position Paper in Rhinosinusitis and Nasal Polyposis) which have critically analysed the existing evidence in the literature and identified the gaps in our knowledge, initiating efforts to fill them.

There has been an increasing recognition of the interplay between the upper and lower respiratory tracts and this has led to initiatives within the European Union to prioritise respiratory disease which includes conditions such as allergy and chronic rhinosinusitis (CRS). The launch of EUFOREA (European Forum for Research and Education in Allergy and Airway diseases) in 2015 marks an important starting point in collaboration at a political level as well as amongst researchers to advance our understanding and management of these diseases and has already resulted in two summits and a number of publications. Whilst our understanding of the epidemiology and pathophysiology of allergic/inflammatory/ infective diseases grows, the importance of personalised or precision medicine, initially espoused in asthma can now be applied to these conditions if the necessary markers can be established to phenotype and endotype them. The increasing availability of clinical trials of new drugs, especially the bio- logics has opened up a range of possible treatments especially in conditions such as CRS with nasal polyps.

There has been an increasing appreciation of the need to actively engage with patients and the public in these initiatives and this can be done using 'apps' which reveal some surprising information about treatment compliance and disease impact.

In the area of neoplasia, epidemiology studies have revealed changes in tumour frequency and we are now seeing the publication of large prospective cohorts of patients with sinonasal malignancies treated endoscopically, allowing robust statistical analysis and comparison with conventional treatments. Finally the increased availability of genomic analysis and stem cell technology are having their impact in the nose and sinuses, opening up exciting possibilities for the future.

\section{References}

1) BROZEK $J$ et al. Allergic Rhinitis and its Impact on Asthma (ARIA) Journal of Allergy and Clinical Immunology 2010 pp1-154.

2) LUND VJ, STAMMBERGER H, NICOLAI P, CASTELNUOVO P et al. (2010) European Position Paper on Endoscopic Management of the Nose, Paranasal Sinuses and Skull Base. Rhinology Supplement 22, pp 1-144.

3) FOKKENS, W, LUND VJ, MULLOL J, BACHERT C, et al European Position paper on rhinosinusitis and nasal polyps 2012 Rhinology Supplement 232012 pp 298. 